\title{
A FERMENTED INFANT MILK FORMULA REDUCES ILEAL PROTEOLYTIC ACTIVITY
}

\author{
S. Huybers, E. Abrahamse, J. Knol, M. Alles, H. Bouritius, T. Ludwig \\ Danone Research - Centre for Specialised Nutrition, Wageningen, The Netherlands
}

Background and aims: Gastrointestinal (GI) proteases are best known for their function in protein digestion. However, GI luminal serine protease activity is also associated with inflammation, pain and visceral hypersensitivity via activation of protease-activated receptor-2 (PAR-2). An infant milk formula comprising product fermented by Streptococcus thermophilus 065 and Bifidobacterium breve C50 has previously been reported to improve gut comfort in infants with digestive problems. Here, the effect of this fermented formula (Lactofidus) on proteolytic activity was investigated at the terminal ileum.

Methods: The fermented formula and a non-fermented standard formula were tested in three-week-old piglets $(\mathrm{N}=6)$ fitted with a $\mathrm{T}$-cannula. Each formula was provided in a Latin square design. Ileal digesta were collected during eight hours and subsequently analyzed for total proteolytic activity and protein digestibility. Proteolytic activity was further characterized either by specific substrates or inhibitors as serine protease, trypsin, or chymotrypsin activity.

Results: Fermented formula yielded a more than threefold lower total proteolytic activity at the terminal ileum compared to standard formula $\left(477 \pm 84 \cdot 10^{3}\right.$ and $1599 \pm 343 \cdot 10^{3}$ units/8h, respectively). This proteolytic activity was found to be mainly constituted by the pancreatic serine proteases trypsin and chymotrypsin. Despite lower proteolytic activity in the fermented formula, protein was fully digested.

Conclusions: The fermented formula causes lower exposure of the small intestine to pancreatic serine proteases. This could contribute to improved gut comfort via diminished activation of PAR-2, which could be particularly beneficial in infants with digestive problems. 